\title{
Propranolol, a $\beta$-adrenergic antagonist, attenuates the decrease in trabecular bone mass in high calorie diet fed growing mice
}

\author{
Kyunghwa Baek ${ }^{1,2}$, Hyo Rin Hwang ${ }^{1}$, Hyun-Jung Park ${ }^{1}$, Arang Kwon ${ }^{1}$, Abdul S. Qadir ${ }^{1}$ E Jeong-Hwa Baek, * \\ ${ }^{1}$ Department of Molecular Genetics, School of Dentistry and Dental Research Institute, Seoul National University, Seoul 110-749, \\ ${ }^{2}$ Department of Pharmacology and Research Institute of Oral Science, College of Dentistry, Gangneung-Wonju National University, Wonju \\ 210-702, Korea
}

\begin{abstract}
We investigated the effects of high calorie and low calorie diets on skeletal integrity, and whether $\beta$-adrenergic blockade (BB) attenuates bone loss induced by dietary calorie alteration. Male 6-week-old $\mathrm{C} 57 \mathrm{BL} / 6$ mice were assigned to either an ad-lib fed control diet (CON), a high calorie diet (HIGH), or a low calorie diet (LOW) group. In each diet group, mice were treated with either vehicle (VEH) or propranolol, a $\beta$-adrenergic antagonist. Over 12-weeks, $\beta$-blockade mitigated body weight and fat mass increases induced by the high calorie diet. Femoral trabecular bone mineral density and the expression levels of osteogenic marker genes in bone marrow cells were reduced in HIGHVEH and LOWVEH mice, and BB significantly attenuated this decline only in HIGH mice. In summary, the magnitude of bone loss induced by low calorie diet was greater than that caused by high calorie diet in growing mice, and $\beta$-blockade mitigated high calorie diet-induced bone loss. [BMB Reports 2014; 47(9): 506-511]
\end{abstract}

\section{INTRODUCTION}

Obesity and obesity-related metabolic disorders have increased rapidly worldwide. Obesity is traditionally known to have beneficial consequences on bone integrity, due to the mechanical loading conferred by body weight. However, it has been recently suggested that increased levels of adipokines or hormones derived from excessively accumulated fat in the context of obesity, could be detrimental to bone (1-3). The underlying pathophysiological mechanism between obesity and bone is very complex, and has not been fully investigated. Meanwhile, in an attempt to lose weight, considerable num-

*Corresponding author. Tel: +82-2-740-8688; Fax: +82-2-741-3193; E-mail: baekjh@snu.ac.kr

http://dx.doi.org/10.5483/BMBRep.2014.47.9.265

Received 28 November 2013, Revised 16 December 2013, Accepted 2 January 2014

Keywords: Beta-adrenergic receptor, Bone mineral density, High calorie diet, Low calorie diet, Propranolol bers of women in modern society restrict dietary calories (4). Chronic restriction of calorie intake can lead to significant clinical concerns, such as decreased bone mass $(5,6)$. On average, each $10 \%$ decrement in body weight in humans is associated with a $1 \%$ decrease in bone mass (7).

Mammalian bones are widely innervated by sympathetic and sensory nerves, which are particularly abundant in regions of high osteogenic activity (8). Over the last decade, a line of research has emerged elucidating the role of the sympathetic nervous system (SNS) in the regulation of bone mass. Osteoblasts express $\beta$-adrenergic receptors (9). SNS activation drives bone loss mainly by reducing osteoblast differentiation and proliferation (9), and by stimulating bone resorption (10). Activation of $\beta$-adrenergic receptors increases the expression of osteolytic factors, such as receptor activator of NF-kappa B ligand, interleukin-6, interleukin-11, and prostaglandin $E_{2}$, as well as the formation of osteoclast-like cells from mouse bone marrow cells (11).

In the context of calorie restriction- or obesity-induced bone loss, a correlation of these phenomena with SNS activation has been suggested. It has been proposed that potential factors contribute to SNS activation in obesity, which include hyperleptinemia, activation of the central pro-opiomelanocortin/melanocortin-4 receptor, hyperinsulinemia/insulin resistance, hypoadiponectinemia, hypoghrelinemia, increased angiotensin II levels, and baroreceptor dysfunction (12). Norepinephrine and/or epinephrine release is also increased with fasting or weight reduction $(13,14)$, and also results in loss of bone mass. Conversely, pharmacological blockade of $\beta$-adrenergic receptors attenuates the loss of trabecular bone in the context of SNS activation-associated stressful conditions, such as disuse via hindlimb unloading in mature rats (15), calorie restriction in adult rats (16), and ovariectomy in exercising adult rats (17). It has also been demonstrated that propranolol, a $\beta$-adrenergic receptor antagonist, attenuates the mechanical unloading-induced or calorie restriction-induced decline in trabecular bone mass, and the bone formation rate in mice $(15,18)$. However, no study has demonstrated whether blocking $\beta$-adrenergic signaling attenuates obesity-induced bone loss, or compared the magnitude of each caloric alteration's (high calorie diet vs. low calorie diet) contributions to bone loss, in a side-by-side study. 
In the present study, we compared the contributions of each dietary calorie alteration (high calorie diet vs. low calorie diet) to bone loss in growing mice, and tested whether $\beta$-adrenergic blockade attenuates decreases in bone mass, during dietary calorie alteration.

\section{RESULTS}

$\beta$-Adrenergic blockade attenuates the increase of body weight and fat mass observed with the high calorie diet

At 12 weeks, body weight and fat mass was lower in LOWVEH mice $(-30.8 \%,-72.83 \%$, respectively), but higher in HIGHVEH mice $(+32.8 \%,+78.79 \%$, respectively), compared to those in CONVEH mice (Figs. 1A and 1B). However, at week 12, the lean body mass was higher in LOWVEH mice $(+25.89 \%)$, but lower in HIGHVEH $(-23.21 \%)$ mice, compared to that in CONVEH mice (Fig. 1C).

Although a significantly higher body fat mass was observed in HIGHBB mice at week $12(+34.05 \%)$, compared to that of CONVEH, the impact of the high calorie diet on body fat mass over 12 weeks was significantly attenuated by propranolol (Fig. 1B). Furthermore, body weight increased over $100 \%$ in HIGHVEH mice at week 12 ; whereas, $\beta$-adrenergic blockade reduced this increase $(+72 \%$ vs. week 0 within group) (Fig. $1 \mathrm{~A})$.

Both the high calorie and low calorie diets reduce femoral trabecular vBMD, but calorie restriction produces greater reduction than the high calorie diet

After 12 weeks, LOWVEH and HIGHVEH mice had lower trabecular vBMD $(-21.21 \%$ and $-16.96 \%$, respectively), and lower trabecular bone volume $(-54.66 \%$ and $-17.84 \%$, respectively), compared to CONVEH mice (Table 1, Fig. 2). Lower trabecular number $(-58.92 \%)$, and higher trabecular separation $(+34.66 \%)$ and porosity $(+3.23 \%)$, were observed only in LOWVEH mice, compared to CONVEH mice.

\section{$\beta$-Adrenergic blockade attenuates the reduction of trabecular femoral vBMD observed with the high calorie diet}

The impact of the high calorie diet on trabecular femoral vBMD reduction was significantly mitigated by propranolol $\left(198.98 \mathrm{mg} / \mathrm{cm}^{3}\right.$ vs. $172.21 \mathrm{mg} / \mathrm{cm}^{3}$ in HIGHVEH mice) (Table 1, Fig. 2). However, no significant differences in $\mu C T$ values, including trabecular femoral $\mathrm{VBMD}$, were observed with propranolol treatment in calorie-restricted animals.

\section{The low calorie diet produces lower cortical thickness and lower MMI in the femoral midshaft}

Following 12 weeks of dietary caloric interventions, although periosteal perimeter $(+3.64 \%)$ and cross-sectional diameter $(+41.85 \%)$ were higher in LOWVEH mice vs. CONVEH mice, cross-sectional bone area $(-14.25 \%)$, cortical thickness $(-12.71 \%)$, and cross-sectional geometry MMI $(-16.37 \%)$ were lower in LOWVEH mice vs. CONVEH mice (Table 1). These data indicate that calorie restricted mice had expanded toward the periosteum, but had thinner, mechanically weaker cortical geometric properties. The impact of the high calorie diet on the cortical bone was observed only in an increased periosteal perimeter $(+3.92 \%$ vs. CONVEH mice). No significant mitigation effects in any $\mu C T$ variables of cortical bone were observed with BB treatment in both LOW and $\mathrm{HIGH}$ animals.

Both the high calorie and low calorie diets induce decreased tibial trabecular bone formation and increased bone resorption, but the low calorie diet produces a greater reduction in the $B F R$

After 12 weeks, both HIGHVEH and LOWVEH mice had significantly lower trabecular bone volume (\% BV/TV, $-17 \%$
(A)

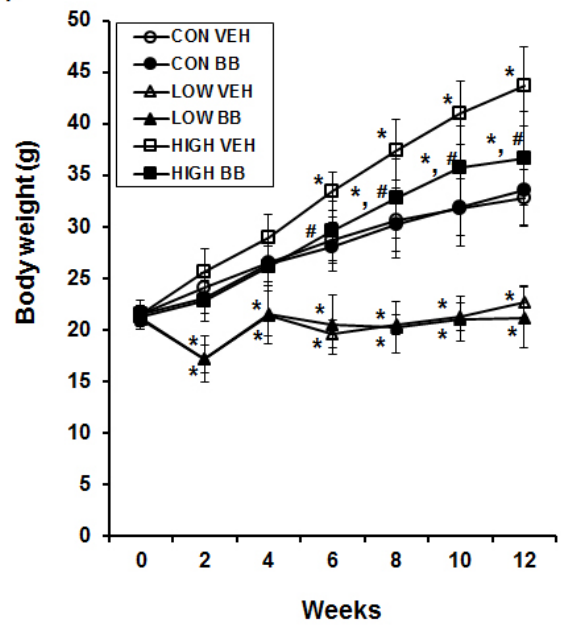

(B)

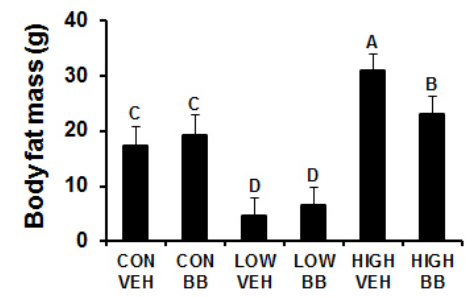

(C)

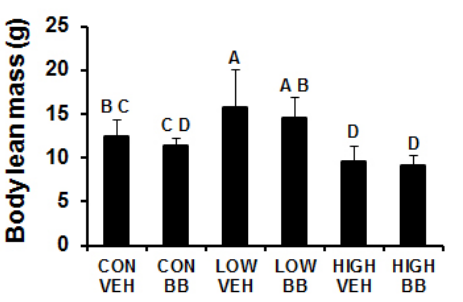

Fig. 1. Blocking $\beta$-adrenergic signaling attenuates the increases in body weight and fat mass observed with the high fat diet. (A) Body weights over 12 weeks of the high fat diet (HF), or the low calorie diet (LOW), or fed ad-lib $(\mathrm{CON})$, in mice administered vehicle (VEH) or $\beta$ blocker (BB). For all experiment groups, $\mathrm{n}=10 .{ }^{*} \mathrm{P}<0.05$ vs. CONVEH, "P $<0.05$ vs. VEH within the same diet group. $(B, C)$ Total body fat mass and lean mass at week 12 , from whole body DEXA scans. Bars sharing the same letters are not significantly different. 


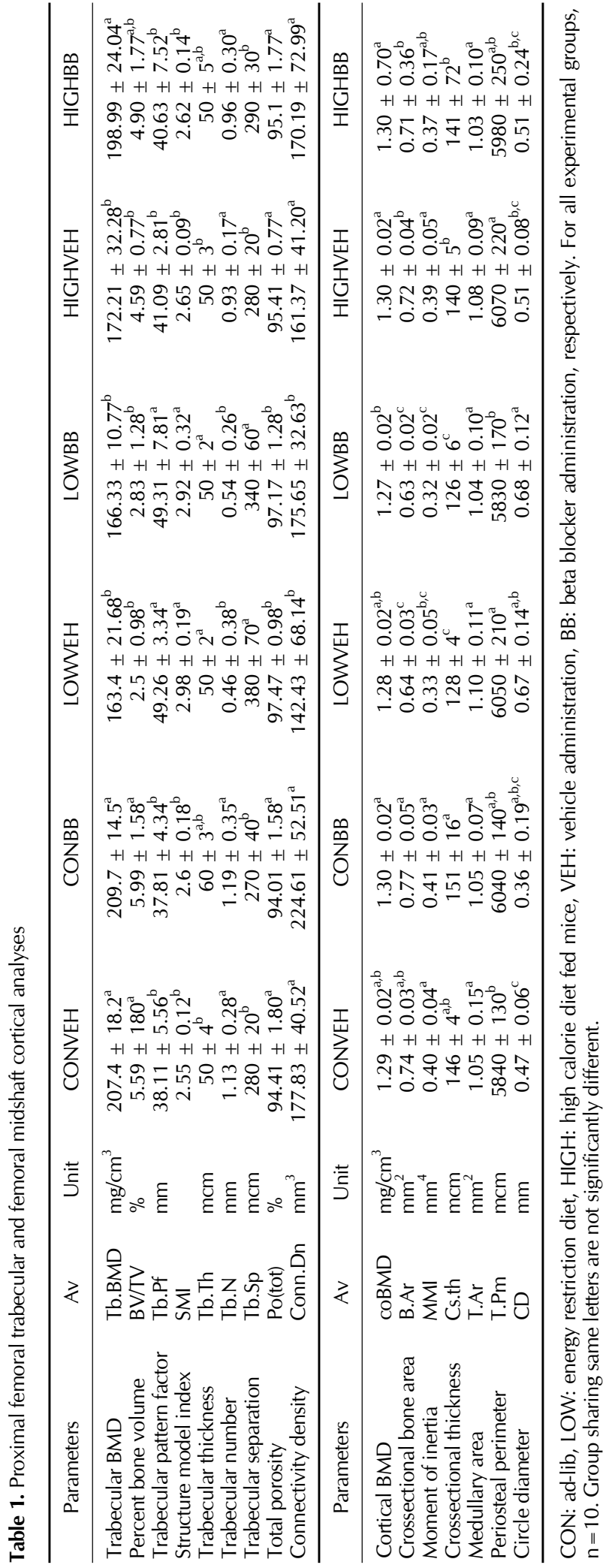

and $-54 \%$, respectively), than that of CONVEH mice (Fig. 2B). The 3-fold decrease in MAR was observed with HIGHVEH mice (vs. CONVEH mice) (Fig. 3A). Although \% MS/BS was numerically lower in HIGHVEH mice, compared to that in CONVEH mice, this reduction was not significant. These reductions in MAR and \% MS/BS contributed to a $60 \%$ reduction in BFR in HIGHVEH mice. The percent eroded surface (the surface covered by osteoclasts) increased approximately 2.8-fold in $\mathrm{HIGH}$ mice vs. CON mice. In calorie-restricted mice, a decrease in both MAR $(-56 \%)$ and \% MS/BS $(-58 \%)$ vs. CONVEH mice was observed. These reductions in MAR and \% MS/BS contributed to the $82 \%$ reduction in BFR in LOWVEH mice. The percent-eroded surface increased by $207 \%$ in LOWVEH mice, and by $181 \%$ in HIGHVEH mice (vs. CONVEH mice).

\section{$\beta$-Adrenergic blockade mitigates the decreased trabecular bone formation and the increased bone resorption observed with the high calorie diet}

The decrease in MAR observed in HIGHVEH mice was blunted by $\beta$ blockade, and this rescued MAR contributed to the attenuation of BFR reduction observed with the high calorie diet (Fig. 3A). The increase in the \%-eroded surface observed in HIGHVEH mice was also abolished in HIGHBB mice. In calorie-restricted mice, propranolol did not exert significant effect on bone formation parameters. However, dramatic increase in the \%-eroded surface observed with the low calorie diet was significantly reversed with $\beta$-adrenergic blockade. In CON mice, propranolol did not induce any significant change in the histomorphometric parameters.

\section{Both the high calorie and low calorie diets suppress} osteogenic marker gene expression in bone marrow cells, and $\beta$-adrenergic blockade attenuates the reduction of osteogenic marker gene expression in the high calorie diet fed diet At week 12, we harvested tibial bone marrow from sacrificed mice, and extracted total RNA. Among the genes examined, the levels of Runx2, Osterix, and BSP mRNA were significantly down-regulated, by both low calorie and high calorie diets (Fig. 3B). The expression level of MEPE was down-regulated only by the high calorie diet. Although the impact of diet alteration on osteogenic gene expression was suppressed by propranolol in both groups, the effect of propranolol was more significant in HIGH mice, than in LOW mice. Among the genes examined, the down-regulation of Osterix and Runx2 mRNA by the low calorie diet was significantly rescued with propranolol treatment. In high calorie diet fed mice, reductions in BSP, MEPE, Osterix, and Runx2 mRNA were significantly rescued with propranolol treatment. Expression of ALP was dramatically increased with $\beta$ blockade, in high calorie diet fed mice. 
(A)

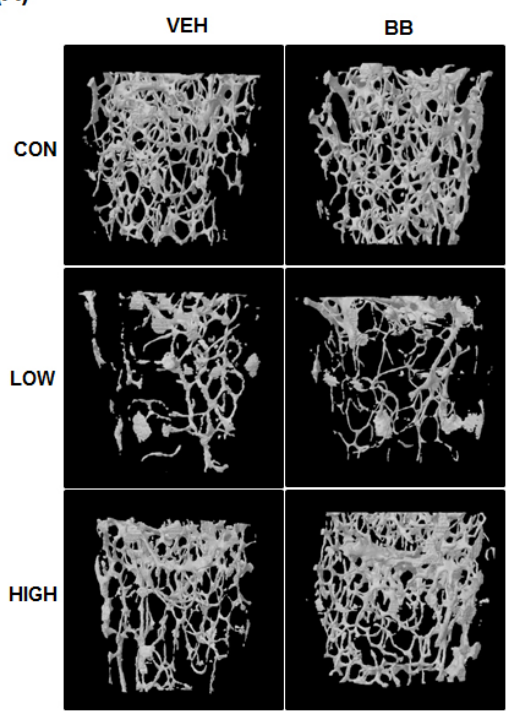

(B)
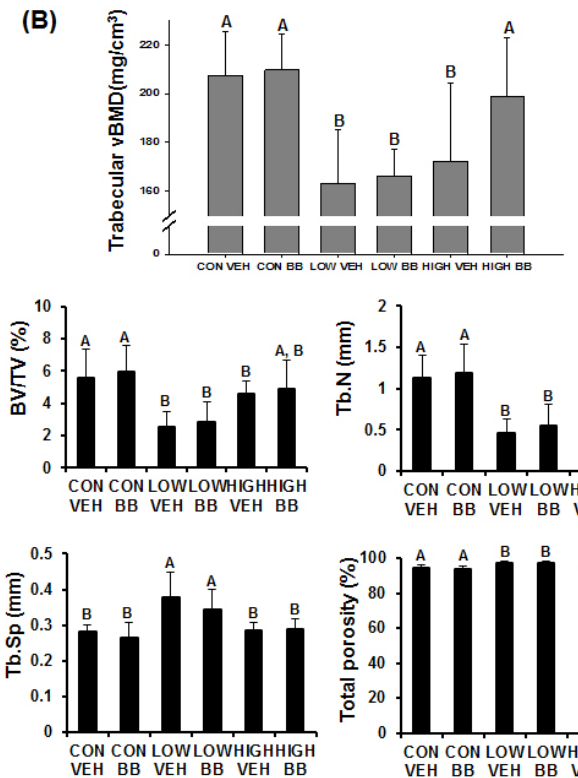
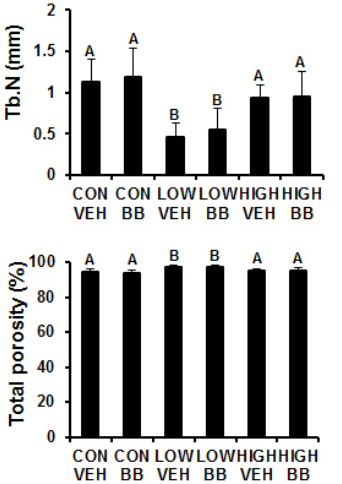

Fig. 2. $\beta$-Adrenergic blockade attenuates the reduction of trabecular femoral vBMD observed with the high calorie diet. Ex vivo $\mu \mathrm{CT}$ assessment of the distal femur was performed, after 12 weeks of dietary alterations, and/or $\beta$ blockade. (A) $\mu \mathrm{A}$ images of cancellous bone at the distal femur. (B) $\mu \mathrm{B}$ analysis results of the cancellous bone density and geometry. For each group, $n=10$. Bars sharing the same letters are not significantly different. Tb.vBMD (Trabecular volumetric BMD), BV/TV (Percent bone volume), Tb.N (Trabecular number), Tb.Sp (Trabecular separation).

(A)
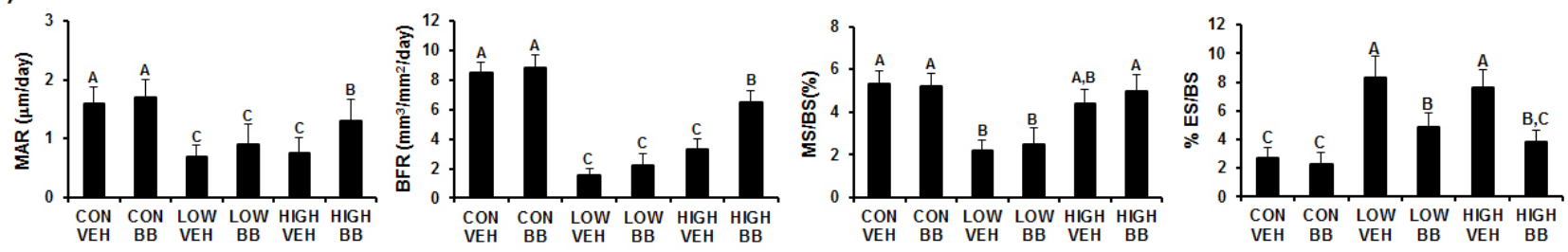

(B)
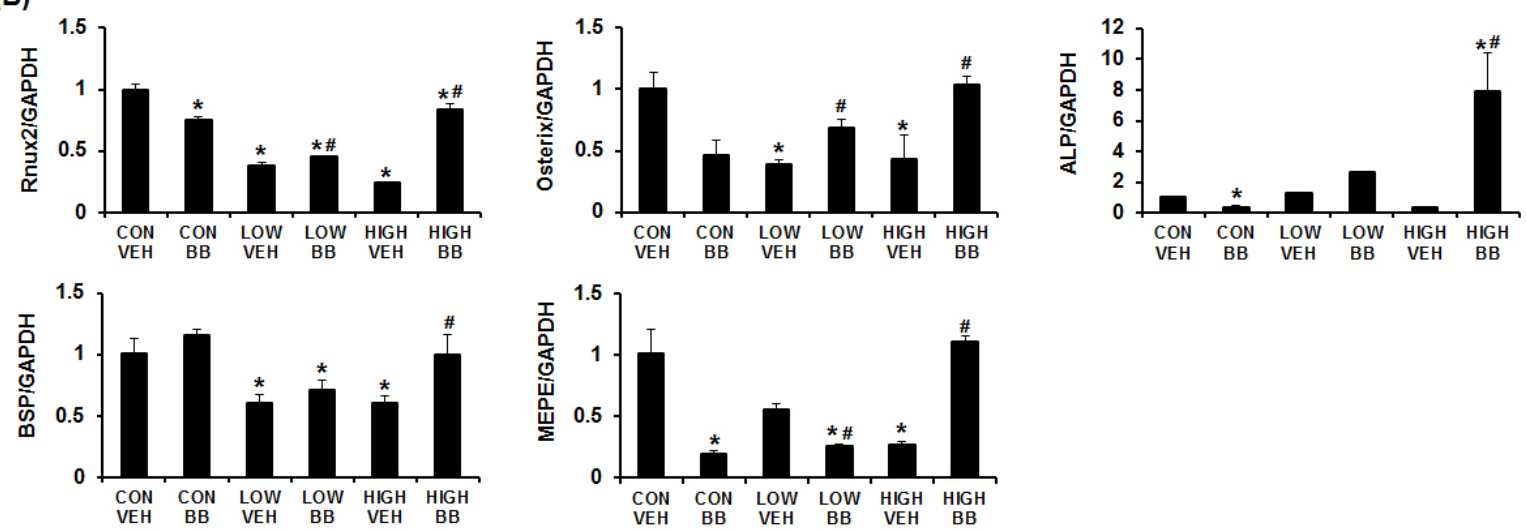

Fig. 3. $\beta$-Adrenergic blockade mitigates the decreased trabecular bone formation and osteogenic marker gene expression observed with the high calorie diet. (A) Histomorphometric analysis results obtained from proximal tibial sections. Data are presented as the mean \pm SD. Bars sharing the same letters are not significantly different. MAR (mineral apposition rate), \% MS/BS (percent mineralizing surface), BFR (bone formation rate), \% ES/BS (percent erosion surface). (B) Tibial bone marrow was harvested after sacrifice, and total RNA was extracted. The mRNA levels of osteogenic marker genes were examined by quantitative RT-PCR. Data are presented as the mean \pm SD of triplicates ( ${ }^{\mathrm{P}}<0.05$ vs. CONVEH, ${ }^{\#} \mathrm{P}<0.05$ vs. VEH within the same diet group). 


\section{DISCUSSION}

These experiments demonstrate the effects of dietary calorie alteration on bone mass and architecture, and the interesting links between the SNS and regulation of bone mass. To our knowledge, this is the first study providing a side-by-side comparison of the effects of low calorie or high calorie diet on bone mass. One of the major objectives of this study was to compare the magnitude of the contribution of each dietary caloric alteration to bone loss. The data showed that both low calorie diet and high calorie diet independently impair the limb bone's skeletal integrity, via decreased bone formation, and increased bone resorption. The detrimental impact of the low calorie diet on the trabecular and cortical femoral bone was significantly greater, than that of the high calorie diet. Reduction of bone volume was observed in both diet groups, but lower trabecular number and higher trabecular space/porosity was observed only with low calorie diet, which reflects this diet's greater deleterious effect on trabecular bone at the metaphyseal site. Additionally, the low calorie and high calorie diets exhibited significant increases in the marrow area (area inside the endocortical perimeter) of the femoral midshaft. Both the low calorie and high calorie diets exhibited a significant decrease in total cross-sectional bone area and cortical thickness. This reflects periosteal expansion/thinness coupled with increased marrow area, suggesting a net loss of cortical bone at this site, and this loss was more exacerbated with the low calorie diet, inducing a lower bone strength index.

We sought to elucidate the effects of moderate calorie restriction on bone outcomes, when all other nutrients were maintained at adequate levels. Comparing our data to those of previously published studies, it is clear that restricting dietary calorie intake causes multiple negative consequences for the geometric properties of bone (6), as well as an increased incidence of uncoupling of bone formation to bone resorption $(18,19)$. In addition to calorie restriction, previous studies have also shown that low bone mass and compromised bone skeletal integrity are associated with diet-induced obesity, in both animals and humans. Growing C57BL/6 mice fed a high-fat diet showed $43 \%$ lower mineral content and 15\% lower bone mineral density in the femur, compared to control diet fed mice (3). High-fat dietary intake during the growth period exerts deleterious effects on bone parameters in mice (2). Changes reported in mice cortical bone in response to a high-fat diet include dramatically lower femoral strength, stiffness, and toughness, compared to controls, from adolescence to adulthood (20).

In the present study, $\beta$-adrenergic blockade exerts its trabecular bone protective effect in the context of high calorie diet, but not in the calorie restricted state in femoral metaphysis. At the femoral midshaft, we observed no significant mitigating effect of $\beta$-adrenergic blockade in cortical bone BMD and geometry, in the low calorie diet fed mice. It was recently re- ported that blocking $\beta$ adrenergic signaling attenuates reductions in trabecular bone mass observed with dietary calorie restriction (18), demonstrating the mitigating effect of $\beta$ blockade in calorie restriction-induced bone loss in adult rodents. However, in that report, skeletally mature rats were used; and the magnitude of the $\beta$ blockade effect in high calorie diet-induced bone loss has not been quantified in a side-by-side comparison. There is a study showing that the detrimental effects of calorie restriction are greater in growing mice, than in mature or aged mice (16). In the present study, it has been shown that the mitigating effect of $\beta$ blockade is not sufficient to rescue the bone loss induced by calorie restriction in growing mice. It is noteworthy, however, that even though they were not significant, $\beta$ blockade suppressed calorie restriction-induced decreases in trabecular VBMD, bone volume, and trabecular number. Additionally, osteogenic marker gene analysis demonstrated that $\beta$-adrenergic blockade mitigates the reduction of Osterix and Runx2 gene expression in bone marrow cells, induced by the low calorie diet.

In summary, both the high calorie and low calorie dietary intake over 12 weeks in growing mice caused significant bone loss in limb bones, and the magnitude of bone loss induced by the low calorie diet was greater, than that of the high calorie diet. $\beta$-Adrenergic blockade mitigated high calorie diet-induced metaphyseal bone loss, by attenuating declines in osteoblastic cell activity, and by abolishing increases in osteoclast surfaces. These data suggest an important role for the $\beta$-adrenergic signaling in bone responses, to alterations in dietary calorie intake.

\section{MATERIALS AND METHODS}

All experimental procedures other than animal experimental design were provided in Supplementary data 1.

\section{Animals and experimental design}

Sixty C57BL/6 mice, aged 5 weeks at purchase (Orient Bio Inc., Seoul, Korea), were block-assigned by body weight into six groups of ten animals each: ad-lib fed controls treated with vehicle (CONVEH) or $\beta$-blocker (CONBB), calorie restricted mice treated with vehicle (LOWVEH) or $\beta$-blocker (LOWBB), and high fat diet mice treated with vehicle (HIGHVEH) or $\beta$-blocker (HIGHBB). The CON group was fed a purified control diet (10\% calories from fat; \#D12450B, Research Diets, New Brunswick, NJ, USA), for the twelve-week protocol. For the high fat diet experiments, mice were fed a high fat diet (60\% calories from fat; \#D12492, Research Diets). To achieve the $30 \%$ calorie restriction, LOW mice were fed $0.7 \mathrm{~g}$ of the specially formulated diet (Research Diets), for every $1 \mathrm{~g}$ of \#D12450B diet of the CONVEH group average consumption. A higher density of other nutrients in the modified diet assured $100 \%$ intake of all nutrients except calories, when a reduced volume of chow was provided. Important vitamin/mineral intakes were adjusted to be equivalent among the three calorie 
groups. Food intake for all mice was measured each day, and within the same calorie groups, $\mathrm{t} 3$ he food intake was not different between VEH and BB mice. $\beta$-Blocker groups were administered propranolol ( \pm )-propranolol hydrochloride, SigmaAldrich, St. Louis, MO, USA) via drinking water (0.5 g/L) (18). On days 9 and 2 before sacrifice, animals were given subcutaneous injections of calcein $(25 \mathrm{mg} / \mathrm{kg}$ ), to label mineralizing bone for histomorphometric analyses. All procedures in this study were approved by the Seoul National University Institutional Animal Care and Use Committee (SNU-110531-2).

\section{ACKNOWLEDGEMENTS}

This work was supported by a National Research Foundation of Korea (NRF) grant, funded by the Korean government (MEST) (2011-0016548).

\section{REFERENCES}

1. Cao, J. J. (2011) Effects of obesity on bone metabolism. J. Orthop. Surg. Res. 6, 30.

2. Lac, G., Cavalie, H., Ebal, E. and Michaux, O. (2008) Effects of a high fat diet on bone of growing rats. Correlations between visceral fat, adiponectin and bone mass density. Lipids Health Dis. 7, 16.

3. Parhami, F., Tintut, Y., Beamer, W. G., Gharavi, N., Goodman, W. and Demer, L. L. (2001) Atherogenic high-fat diet reduces bone mineralization in mice. J. Bone Miner. Res. 16, 182-188.

4. Yaemsiri, S., Slining, M. M. and Agarwal, S. K. (2011) Perceived weight status, overweight diagnosis, and weight control among US adults: the NHANES 2003-2008 Study. Int. J. Obes. (Lond) 35, 1063-1070.

5. Ihle, R. and Loucks, A. B. (2004) Dose-response relationships between energy availability and bone turnover in young exercising women. J. Bone Miner. Res. 19, 12311240.

6. Talbott, S. M., Cifuentes, M., Dunn, M. G. and Shapses, S. A. (2001) Energy restriction reduces bone density and biomechanical properties in aged female rats. J. Nutr. 131, 2382-2387.

7. Riedt, C. S., Cifuentes, M., Stahl, T., Chowdhury, H. A., Schlussel, Y. and Shapses, S. A. (2005) Overweight postmenopausal women lose bone with moderate weight reduction and $1 \mathrm{~g}$ /day calcium intake. J. Bone Miner. Res. 20, 455-463.

8. Bjurholm, A. (1991) Neuroendocrine peptides in bone. Int. Orthop. 15, 325-329.
9. Takeda, S., Elefteriou, F., Levasseur, R., Liu, X., Zhao, L., Parker, K. L., Armstrong, D., Ducy, P. and Karsenty, G. (2002) Leptin regulates bone formation via the sympathetic nervous system. Cell 111, 305-317.

10. Elefteriou, F., Ahn, J. D., Takeda, S., Starbuck, M., Yang, X., Liu, X., Kondo, H., Richards, W. G., Bannon, T. W., Noda, M., Clement, K., Vaisse, C. and Karsenty, G. (2005) Leptin regulation of bone resorption by the sympathetic nervous system and CART. Nature 434, 514-520.

11. Takeuchi, T., Tsuboi, T., Arai, M. and Togari, A. (2001) Adrenergic stimulation of osteoclastogenesis mediated by expression of osteoclast differentiation factor in MC3T3-E1 osteoblast-like cells. Biochem. Pharmacol. 61, 579-586.

12. da Silva, A. A., do Carmo, J., Dubinion, J. and Hall, J. E. (2009) The role of the sympathetic nervous system in obesity-related hypertension. Curr. Hypertens. Rep. 11, 206211.

13. Trayhurn, P., Hoggard, N., Mercer, J. G. and Rayner, D. V. (1999) Leptin: fundamental aspects. Int. J. Obes. Relat. Metab. Disord. 23 (Suppl 1), 22-28.

14. Zauner, C., Schneeweiss, B., Kranz, A., Madl, C., Ratheiser, K., Kramer, L., Roth, E., Schneider, B. and Lenz, K. (2000) Resting energy expenditure in short-term starvation is increased as a result of an increase in serum norepinephrine. Am. J. Clin. Nutr. 71, 1511-1515.

15. Baek, K. and Bloomfield, S. A. (2009) Beta-adrenergic blockade and leptin replacement effectively mitigate disuse bone loss. J. Bone Miner. Res. 24, 792-799.

16. Tatsumi, S., Ito, M., Asaba, Y., Tsutsumi, K. and Ikeda, K. (2008) Life-long caloric restriction reveals biphasic and dimorphic effects on bone metabolism in rodents. Endocrinology 149, 634-641.

17. Bonnet, N., Beaupied, H., Vico, L., Dolleans, E., Laroche, N., Courteix, D. and Benhamou, C. L. (2007) Combined effects of exercise and propranolol on bone tissue in ovariectomized rats. J. Bone Miner. Res. 22, 578-588.

18. Baek, K. and Bloomfield, S. A. (2012) Blocking beta-adrenergic signaling attenuates reductions in circulating leptin, cancellous bone mass, and marrow adiposity seen with dietary energy restriction. J. Appl. Physiol. 113, 1792-1801.

19. Banu, M. J., Orhii, P. B., Mejia, W., McCarter, R. J., Mosekilde, L., Thomsen, J. S. and Kalu, D. N. (1999) Analysis of the effects of growth hormone, voluntary exercise, and food restriction on diaphyseal bone in female F344 rats. Bone 25, 469-480.

20. Ionova-Martin, S. S., Wade, J. M., Tang, S., Shahnazari, M., Ager, J. W., 3rd, Lane, N. E., Yao, W., Alliston, T. Vaisse, C. and Ritchie, R. O. (2011) Changes in cortical bone response to high-fat diet from adolescence to adulthood in mice. Osteoporos Int. 22, 2283-2293. 